\title{
Posicionamento das alças de lentes intraoculares implantadas intencionalmente no sulco ciliar através da biomicroscopia ultrassônica
}

\author{
Positioning of intraocular lens haptics intentionally implanted in the ciliary sulcus \\ by ultrasound biomicroscopy
}

Ricardo Rau ${ }^{1}$, Carmen Resende Santana ${ }^{1}$, Andrea Alejandra Gonzalez Martinez ${ }^{1}$, André Luiz de Freitas Silva ${ }^{1}$, Norma Allemann ${ }^{1}$

\section{RESUMO}

Objetivos: Avaliar a posição das alças das lentes intraoculares implantadas intencionalmente no sulco ciliar, em olhos submetidos à cirurgia de catarata com complicação de ruptura de cápsula posterior, e correlacionar os achados com alterações clínicas observadas no exame oftalmológico, utilizando a biomicroscopia ultrassônica.

Métodos: Onze olhos (11 pacientes) que apresentaram ruptura de cápsula posterior durante a cirurgia de catarata com implantação intencional das alças no sulco ciliar foram submetidos ao exame oftalmológico e biomicroscopia ultrassônica. Biomicroscopia ultrassônica avaliou os seguintes parâmetros: posicionamento da porção distal das alças, inclinação e descentração da lente intraocular. O exame oftalmológico foi focalizado para avaliar a presença de "flare" e células na câmara anterior, depósitos na lente e defeitos de transiluminação de íris. A pressão intraocular foi medida, a pigmentação do trabeculado foi determinada, e a avaliação fundoscópica foi necessária para afastar a presença de ruptura retiniana periférica e edema de mácula.

Resultados: Tempo pós-operatório médio para os exames: 103,09 \pm 32,93 dias. Assimetria da posição foi observada em 3 olhos (27,2\%), que tinham alça no sulco ciliar e a segunda alça localizada na pars plana em 2 olhos, associada à inclinação e descentração da lente intraocular; ou no corpo ciliar (1 olho). O exame oftalmológico observou: 5 (45,5\%) olhos com defeitos de transiluminação de íris, 2 (18,1\%) olhos com descentração da lente intraocular; 1 olho (9\%) apresentou hipertensão intraocular. Em todos os casos observou-se hiperpigmentação do trabeculado à gonioscopia. Nenhum caso de rotura periférica de retina e/ou edema de mácula foi relatado.

Conclusões: A biomicroscopia ultrassônica foi capaz de localizar as alças das lentes intraoculares implantadas intencionalmente no sulco ciliar durante cirurgia complicada de catarata, e pôde demonstrar a relação da descentração da lente intraocular com a implantação assimétrica das alças.

Descritores: Implante de lente intraocular; Extração de catarata; Catarata; Ultrassonografia

\begin{abstract}
Purpose: To evaluate the position of haptics of intraocular lens intentionally implanted in the ciliary sulcus in eyes undergoing cataract surgery complication associated with intraoperative posterior capsule rupture, as well as to correlate the findings with clinical changes observed in ophthalmic examination, utilizing ultrasound biomicroscopy

Methods: Eleven eyes (11 patients) who had posterior capsule rupture during cataract surgery with intentional implantation of the haptics in the ciliary sulcus, underwent complete ophthalmic examination and ultrasound biomicroscopy. Ultrasound biomicroscopy evaluated the parameters: positioning of the distal portion of the haptics, tilt and decentration of the intraocular lens. Ophthalmic examination was aimed to evaluate the presence of flare and cells in the anterior chamber, deposits on the lens and iris transillumination defects. Intraocular pressure was measured, pigmentation of the trabecular meshwork was determined, and a fundoscopic evaluation was needed to rule out peripheral retinal rupture and macular edema.

Results: Mean postoperative timefor theexaminations: $103.09 \pm 32.93$ days. Asymmetry of the haptics positioning was observed in 3 eyes (27.2\%) that had one haptic in the ciliary sulcus, the second haptic was placed in the pars plana in 2 eyes, associated to intraocular lens tilt and decentration; or in the ciliary body (1 eye). Ophthalmic examination observed: 5 (45.5\%) eyes with iris transillumination defects, 2 (18.1\%) with intraocular lens decentration; 1 eye (9\%) presented ocular hypertension. In all cases trabecular hyperpigmentation was observed at gonioscopy. No cases of peripheral retinal rupture and/or macular edema were reported.

Conclusions: Ultrasound biomicroscopy was able to locate the intraocularlens haptics intentionally implanted in the ciliary sulcus during complicated cataract surgery, and could demonstrate the relation of intraocular lens decentration to assymetric haptic implantation
\end{abstract}

Keywords: Lens, implantation, intraocular; Cataract extraction; Cataract; Ultrasonography

\section{INTRODUÇÃO}

A rotura de cápsula posterior (RCP) é uma complicação intraoperatória importante na cirurgia de catarata, principalmente entre os cirurgiões iniciantes. O implante primário da lente intraocular (LIO) de câmara posterior no sulco ciliar continua sendo uma opção para reabilitação visual em olhos com suporte capsular periférico adequado ${ }^{(1-3)}$

Estudos já descreveram complicações associadas ao implante da LIO no sulco ciliar, sendo a dispersão de pigmento iriano, associada com defeitos de transiluminação da íris, a mais comum ${ }^{(4)}$. A elevação da pressão intraocular (PIO) e sintomas causados pela borda da LIO, secundários à descentração da mesma, também foram observados em vários pacientes. Outros achados, como hemorragia intraocular e edema macular cistoide, já foram relatados com menor frequência ${ }^{(5)}$. Em muitos casos, para se resolver a complicação, faz-se necessário um procedimento cirúrgico. No estudo de Chang et al., relativo ao implante de LIO acrílica peça única no sulco, a intervenção cirúrgica para reposicionamento ou troca de LIO foi necessária em 93,0\% dos olhos, que apresentaram complicações como sintomas causados pela borda da LIO, aumento da $\mathrm{PIO}$ e hemorragia intraocular ${ }^{(5)}$.
Submetido para publicação: 21 de junho de 2012

Aceito para publicação: 10 de março de 2013

Trabalho realizado no Departamento de Oftalmologia, Universidade Federal de São Paulo - UNIFESP. Médico, Departamento de Oftalmologia, Universidade Federal de São Paulo - UNIFESP - São Paulo (SP), Brasil.
Financiamento: Não houve financiamento para este trabalho.

Declaração de Conflitos de interesses: R.Rau, Nenhum; C.R.Santana, Nenhum; A.A.G.Martinez, Nenhum; A.L.F.Silva, Nenhum; N.Allemann, Nenhum.

Endereço para correspondência: Norma Allemann. Rua Olimpíadas, 134 - Conj. 51 - São Paulo (SP) - 04551-000 - Brasil - E-mail: norma.allemann@pobox.com 
Durante o procedimento cirúrgico de implantação da LIO no sulco ciliar, em muitos casos é difícil determinar com precisão o posicionamento correto das alças. A possibilidade de localização assimétrica entre as alças é bastante alta ${ }^{(6,7)}$.

A biomicroscopia ultrassônica (UBM) permite o exame da câmara posterior e a avaliação da posição das alças da LIO, avaliando a sua proximidade com o sulco ciliar, íris e corpo ciliar ${ }^{(8,9)}$.

O objetivo deste estudo é, através da UBM, avaliar a localização das alças das LIO implantadas intencionalmente no sulco ciliar, em olhos submetidos à cirurgia de catarata associada à complicação intraoperatória de RCP, assim como correlacionar os achados com as alterações clínicas observadas no exame oftalmológico.

\section{MÉTODOS}

Trata-se de um estudo observacional de uma série de casos de pacientes submetidos à cirurgia de catarata pela técnica de facoemulsificação com a complicação de ruptura de cápsula posterior (RCP) e nos quais a LIO foi implantada intencionalmente no sulco ciliar. Os dados foram coletados entre o período de julho de 2011 a fevereiro de 2012 e os pacientes que aceitaram participar do estudo, aprovado pelo Comitê de Ética e Pesquisa da Universidade Federal de São Paulo, sob o número CEP 0062/11; assinaram o termo de consentimento livre e esclarecido e realizaram o exame de UBM e a avaliação oftalmológica completa. O estudo aderiu aos princípios da Declaração de Helsinki.

A técnica cirúrgica compreendeu o procedimento de facectomia por facoemulsificação (equipamento Legacy ${ }^{\circledR}$ ou Infinity ${ }^{\circledR}$, Alcon Inc.), com incisão em córnea clara temporal superior ou nasal superior (preferência de cada cirurgião), aspiração de córtex, e após a identificação da complicação de ruptura da cápsula posterior, também a vitrectomia anterior automatizada, e posteriormente, o implante da LIO no sulco ciliar, durante o mesmo procedimento.

Como critério de inclusão, os pacientes selecionados tinham diagnóstico de catarata senil e período de pós-operatório acima de 60 dias. Como critérios de exclusão foram considerados casos de catarata de etiologia secundária, casos de deiscência zonular (descrita pelo cirurgião no relatório cirúrgico), casos em que a cirurgia de catarata foi associada a outro procedimento cirúrgico (trabeculectomia, vitrectomia programada) e casos com cirurgia intraocular prévia no olho estudado. Na data do exame de UBM os olhos não apresentavam utilização de medicação tópica que pudesse influenciar na posição da íris durante o exame.

Os exames de UBM foram realizados por um único profissional experiente (AAGM), utilizando o aparelho UBM VUMAX $\|^{\circledR}$, Sonomed, que possui um transdutor de $50 \mathrm{MHz}$, com penetração de $13 \mathrm{~mm}$ e excursão de 14 a $16 \mathrm{~mm}$. Os pacientes adotaram a posição de decúbito dorsal horizontal e o exame foi realizado em ambiente iluminado, para simular a miose fisiológica. O exame foi realizado após instilação de colírio anestésico (Anestalcon ${ }^{\circledR}$, Alcon), colocação de blefarostato de acrílico entre as pálpebras, que funcionou também como recipiente para contenção de soro fisiológico a 0,9\% para realização do banho de imersão. O transdutor de $50 \mathrm{MHz}$ é externo, realiza uma excursão de vai-e-vem para promover a varredura e é introduzido no meio líquido que permite a transmissão das ondas sonoras ao olho. Depois de finalizado o exame, as imagens foram selecionadas a partir dos "videoclips" arquivados no equipamento e as medidas foram realizadas utilizando-se o recurso "caliper" do software do aparelho.

Nas imagens obtidas com UBM foram analisados os seguintes parâmetros:

- distância entre a face endotelial da córnea e a face anterior da porção óptica da LIO (DEL) em mm, na área central, no corte axial;

- abertura do ângulo da câmara anterior (ACA), que é formada pela área tangente à rede trabecular e a tangente à superfície anterior da íris, medida a partir do vértice do recesso da íris, em graus, no corte radial ou longitudinal, nos meridianos de 12,3, 6 e 9 horas:

- distância entre a borda da porção óptica da LIO e a face posterior da íris (DIL): para avaliação da inclinação da lente, tendo sido considerada inclinada quando a diferença entre as duas bordas da lente naquele corte e o plano da íris foi superior a 100 micra $(0,10 \mathrm{~mm})$, medida realizada, no corte axial, nos eixos horizontal (meridianos de 3 e 9 horas) e vertical (meridianos de 12 e 6 horas) $)^{(6)}$.

- localização da porção distal de cada uma das alças em relação a referências anatômicas do segmento posterior, com as seguintes possibilidades: sulco ciliar, saco capsular, corpo ciliar (pars plicata) e pars plana; em cortes radiais ou longitudinais.

No exame oftalmológico realizado em uma segunda consulta, foram avaliados: melhor acuidade visual com correção; biomicroscopia do segmento anterior: presença de "flare" e de células na câmara anterior, defeitos de transiluminação de íris, dispersão de pigmento no ângulo (gonioscopia com lente de 4 espelhos), depósito de pigmentos na LIO, e descentração da LIO em midríase; aferição da PIO com tonômetro de Goldmann, sendo considerada elevada quando maior que $21 \mathrm{mmHg}$; e avaliação do segmento posterior: presença de edema macular cistóide e de rotura retiniana periférica utilizando-se oftalmoscopia indireta com lente de 20 D e biomicroscopia de fundo com lente de $78 \mathrm{D}$.

O método estatístico utilizado foi a média aritmética simples.

\section{RESULTADOS}

Foram identificados 16 pacientes submetidos à facectomia por facoemulsificação que apresentaram RCP e nos quais as alças da LIO foram intencionalmente implantadas no sulco ciliar, mas 5 pacientes não aceitaram participar do estudo devido à distância entre seu domicílio e o local dos exames. O estudo foi realizado com 11 olhos de 11 pacientes (nove mulheres e dois homens).

A tabela 1 mostra os dados demográficos dos participantes. A média de idade foi de $75 \pm 9$ anos, um grupo considerado homogêneo. O tempo médio entre a cirurgia e o exame de UBM foi de 103,09 $\pm 32,93$ dias e os olhos não se apresentavam em uso de medicação tópica. As lentes implantadas nesta amostra foram lentes pseudofácicas de câmara posterior: de três peças (modelos TYPE7B ${ }^{\circledR}$ e MA60AC ${ }^{\circledR}$, Alcon, Inc. e modelo Matrix Acrylic $401{ }^{\circledR}$, Medennium Inc.); e lentes de peça única (modelo SLIM ${ }^{\circledR}$, Mediphacos Inc.). O poder dióptrico médio das lentes implantadas foi $+21,72 \pm 1,66$ dioptrias (Tabela 1).

A tabela 2 mostra os achados no exame de UBM. A média da distância central entre face endotelial da córnea e a LIO foi de 3,60 \pm $0,37 \mathrm{~mm}$. A média da distância entre a borda da lente e a face posterior da íris foi de 0,33 $\pm 0,20 \mathrm{~mm}$. A média da abertura do ângulo da câmara anterior foi de 40,71 $\pm 6,72$ graus. Observou-se inclinação e descentração da LIO em dois pacientes (18,1\%). Em destaque na tabela, observa-se que o paciente 3 apresenta uma diferença entre a DIL no meridiano vertical (medidas de 0,2 $\mathrm{mm}$ no meridiano de $12 \mathrm{~h}$ e 1,00 $\mathrm{mm}$ no meridiano de $6 \mathrm{~h}$ ) superior a $0,10 \mathrm{~mm}$, caracterizando a inclinação da LIO. O mesmo ocorre com o paciente 8, no meridianos horizontal (medidas de 0,75 mm no meridiano de $3 \mathrm{~h}$ e 0,15 $\mathrm{mm}$ no meridiano de 9 h). Em 7 (63,6\%) olhos, pôde-se observar contato da LIO com a porção pupilar da íris.

Na tabela 3, que mostra a localização das alças das lentes, pode-se observar que em $3(27,2 \%)$ olhos ocorreu assimetria do posicionamento das alças: $2(18,1 \%)$ olhos com uma alça no sulco ciliar e a outra alça na pars plana e 1 (9\%) olho com uma alça no sulco e a outra no corpo ciliar (Figura 1). Nos 8 (72,7\%) olhos restantes, ambas as alças estavam simetricamente posicionadas no sulco ciliar.

A tabela 4 mostra os achados do exame oftalmológico. Na biomicroscopia em lâmpada de fenda não foi observada a presença de "flare" e/ou células na câmara anterior em nenhum dos olhos. As alterações irianas observadas em 5 (45,5\%) olhos foram decorrentes 
Tabela 1. Dados demográficos pré-operatórios dos participantes submetidos a implante de lente intraocular (LIO) no sulco ciliar durante cirurgia de catarata

\begin{tabular}{ccccccccc}
\hline Paciente & Sexo & $\begin{array}{c}\text { Idade } \\
\text { (anos) }\end{array}$ & Olho & $\begin{array}{c}\text { Seguimento } \\
\text { (dias) }\end{array}$ & $\begin{array}{c}\text { Graduação } \\
\text { catarata }\end{array}$ & $\begin{array}{c}\text { AV corrigida } \\
\text { pré-operatória }\end{array}$ & $\begin{array}{c}\text { Modelo LIO } \\
\text { selecionado }\end{array}$ & $\begin{array}{c}\text { PD LIO } \\
\text { selecionada }\end{array}$ \\
\hline 1 & M & 59 & D & 106 & N1+/SCP2+ & $20 / 80$ & TYPE7B & +19.5 \\
2 & $F$ & 70 & E & 105 & N2+ & $20 / 80$ & TYPE7B & +21.5 \\
3 & $F$ & 72 & D & 61 & N3+ & $20 / 100$ & Mediphacos SLIM & +20.5 \\
4 & M & 86 & D & 64 & N3+ & $20 / 100$ & TYPE7B & +20.0 \\
5 & F & 62 & D & 73 & N2+/SCP1+ & $20 / 80$ & MA60AC & +25.0 \\
6 & F & 79 & E & 143 & N2+ & $20 / 70$ & Matrix Acrylic & +22.0 \\
7 & F & 74 & D & 112 & N2+/SCP3+ & $20 / 100$ & Mediphacos SLIM & +21.5 \\
8 & F & 75 & E & 135 & N1+ & $20 / 60$ & Mediphacos SLIM & +24.0 \\
9 & F & 82 & D & 120 & N1+/SCP1+ & $20 / 50$ & TYPE7B & +22.5 \\
10 & F & 86 & E & 65 & N2+/CA2+ & $20 / 60$ & TYPE7B & +20.5 \\
11 & F & 81 & D & 150 & N2+ & $20 / 70$ & Mediphacos SLIM & +22.0 \\
\hline
\end{tabular}

$\mathrm{M}=$ masculino; $\mathrm{F}=$ feminino; $\mathrm{D}=$ direito; $\mathrm{E}=$ esquerdo; $\mathrm{N}=$ nuclear; $\mathrm{SCP}=$ subcapsular posterior; $\mathrm{CA}=$ cortical anterior; $\mathrm{PD}=$ poder dióptrico; $\mathrm{AV}=$ acuidade visual.

Tabela 2. Parâmetros analisados pela biomicroscopia ultrassônica em olhos submetidos a implante de lente intraocular (LIO) no sulco ciliar durante cirurgia de catarata

\begin{tabular}{|c|c|c|c|c|c|c|c|c|c|c|}
\hline \multirow[b]{2}{*}{ Paciente } & \multirow[b]{2}{*}{ DEL (mm) } & \multicolumn{4}{|c|}{ DIL (mm) } & \multicolumn{4}{|c|}{ ACA (graus) } & \multirow{2}{*}{$\begin{array}{c}\text { Posição das alças } \\
\text { (meridiano horário) }\end{array}$} \\
\hline & & $12 \mathrm{~h}$ & $3 h$ & $6 h$ & $9 h$ & $12 \mathrm{~h}$ & $3 h$ & $6 h$ & $9 h$ & \\
\hline 1 & 4,08 & 0,37 & 0,48 & 0,46 & 0,54 & 44,70 & 49,90 & 47,70 & 45,70 & 1 e 7 \\
\hline 2 & 3,37 & 0,19 & 0,00 & 0,21 & 0,00 & 48,10 & 0,00 & 49,50 & 33,10 & 11 e 5 \\
\hline 3 & 3,48 & 0,20 & 0,40 & 1,00 & 0,41 & 45,80 & 43,90 & 44,90 & 41,60 & 6 e 12 \\
\hline 4 & 4,00 & 0,56 & 0,60 & 0,54 & 0,66 & 32,30 & 43,20 & 34,60 & 40,90 & 6 e 12 \\
\hline 5 & 3,33 & 0,12 & 0,00 & 0,11 & 0,00 & 43,20 & 47,60 & 46,60 & 43,30 & 3 e 9 \\
\hline 6 & 3,37 & 0,16 & 0,16 & 0,16 & 0,16 & 42,50 & 42,80 & 40,30 & 38,60 & 2 e 8 \\
\hline 7 & 3,58 & 0,36 & 0,26 & 0,41 & 0,30 & 44,70 & 47,10 & 46,80 & 46,20 & 6 e 12 \\
\hline 8 & 3,04 & 0,35 & 0,75 & 0,40 & 0,15 & 29,30 & 0,00 & 30,10 & 38,50 & 4 e 10 \\
\hline 9 & 3,75 & 0,11 & 0,12 & 0,13 & 0,12 & 36,90 & 44,50 & 41,90 & 48,00 & 6 e 12 \\
\hline 10 & 4,24 & 0,64 & 0,56 & 0,71 & 0,58 & 40,30 & 45,20 & 40,40 & 46,50 & 1 e 7 \\
\hline 11 & 3,33 & 0,26 & 0,29 & 0,27 & 0,26 & 40,00 & 47,70 & 45,10 & 45,10 & 3 e 9 \\
\hline
\end{tabular}

$\mathrm{DEL}=$ distância entre a face endotelial da córnea e LIO; DIL= distância entre a face posterior da íris e LIO; ACA= ângulo da câmara anterior.

Tabela 3. Localização relativa das alças de cada lente intraocular detectada pela UBM em olhos submetidos a implante de lente intraocular no sulco ciliar durante cirurgia de catarata. Número total de olhos incluídos no estudo: 11, 22 alças de lente intraocular

\begin{tabular}{lccccc}
\hline & & \multicolumn{3}{c}{ Posicionamento da Alça 2} \\
\cline { 2 - 5 } Posicionamento da Alça 1 & Sulco ciliar & Pars plicata & Pars plana & Saco capsular & Total \\
\hline Sulco ciliar & $8(72,72)$ & $1(9,09)$ & $2(18,18)$ & 0 \\
\hline
\end{tabular}

do trauma cirúrgico e caracterizaram-se por áreas localizadas de atrofia do bordelete ou do estroma iriano. A descentração da LIO foi observada em 2 (18,1\%) olhos (Figura 2). A PIO média encontrada na série foi de $15 \pm 2 \mathrm{mmHg}$, sendo que um olho necessitou de medicação antiglaucomatosa para controle da mesma. Em todos os olhos ( $n=11)$ foi observada uma hiperpigmentação do trabeculado, à gonioscopia. Na avaliação de fundoscopia, não foram observados rotura periférica de retina e/ou edema de mácula.

\section{DISCUSSÃO}

O implante da LIO no sulco ciliar nos olhos que apresentam RCP, onde há um suporte capsular periférico adequado, é um procedi- mento bastante frequente, principalmente em centros de ensino de cirurgia de catarata. Determinar com exatidão o posicionamento das alças no sulco ciliar é muito difícil durante a cirurgia.

Assim como no presente estudo, que demonstrou assimetria das alças em $3(27,2 \%)$ olhos, outros autores também relataram com frequência a assimetria da posição das alças nestas situações, como Loya et al. ${ }^{(6)}$, que em seu estudo com 36 olhos (36 pacientes) submetidos à facectomia extracapular complicada com RCP, observaram 15 (42,0\%) olhos com assimetria das alças: uma implantada no sulco ciliar e a outra no saco capsular, pars plicata ou pars plana. Vasavada et al. ${ }^{(4)}$, em estudo com 10 olhos submetidos à facectomia por facoemulsificação e implante de LIO acrílica de peça única no sulco ciliar, também observaram assimetria das alças em 3 (30,0\%) olhos. 


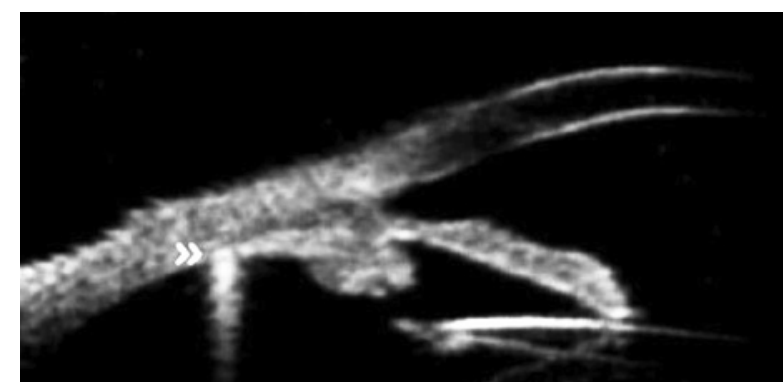

A
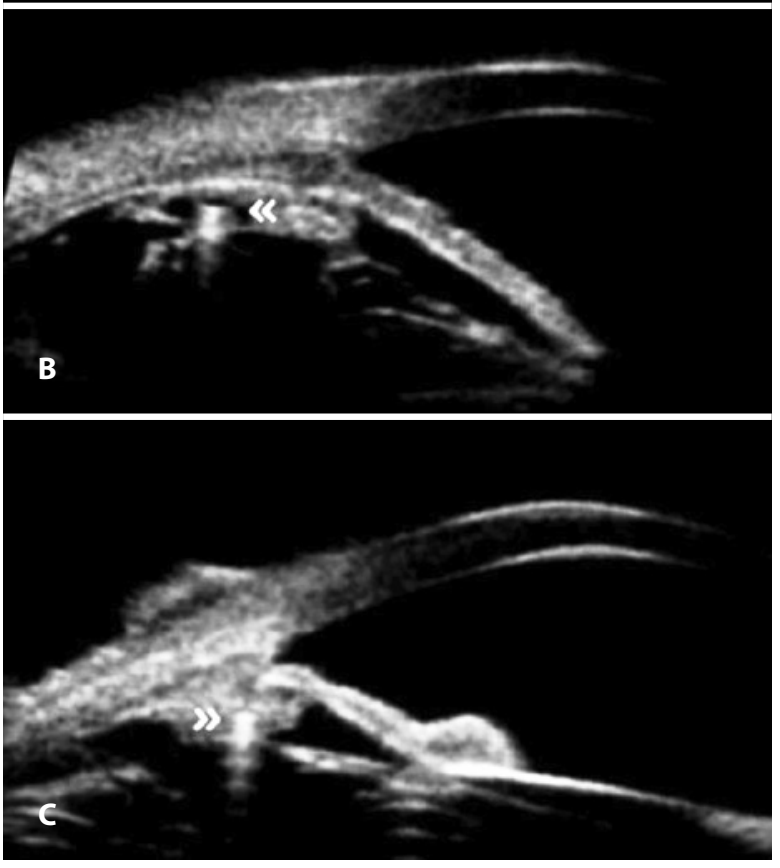

Figura 1.Biomicroscopia ultrassônica (ultrassonografia de $50 \mathrm{MHz}$ ) de olhos submetidos a implante de lente intraocular pseudofácica no sulco ciliar. A alça é identificada como uma estrutura cilíndrica de alta refletividade que causa um artefato de reverberação (duplicação de ecos) disposto posteriormente. A) Paciente 8, demonstra a alça localizada posteriormente aos processos ciliares, na pars plana (seta); B) Paciente 3, alça localizada posteriormente na pars plana (seta); C) Paciente 2, alça localizada sobre a pars plicata, ou seja, sobre os processos ciliares (seta).
Amino e Yamakawa(7) também relataram, após facectomia por facoemulsificação e implante de LIO de diferentes fabricantes, assimetria em 3 (15,7\%) dos 19 olhos estudados, sendo que nos três casos, a segunda alça estava no corpo ciliar.

As medidas de DEL, DIL e ACA encontradas foram, respectivamente, de 3,60 $\pm 0,37 \mathrm{~mm}, 0,33 \mathrm{~mm} \pm 0,20 \mathrm{~mm}$ e 40,71 \pm 6,72 graus, semelhantes às reportadas no estudo de Vasavada et al. ${ }^{(4)}: 3,47 \pm$ $0,24 \mathrm{~mm}, 0,16 \pm 0,07 \mathrm{~mm}$ e 40,2 \pm 4,5 graus, respectivamente.

Utilizando o mesmo critério empregado no presente estudo, para a determinação de inclinação da LIO no exame de UBM, Loya et al. ${ }^{(6)}$ relataram $20(56,0 \%)$ olhos que apresentaram inclinação da LIO, enquanto Vasavada et al. ${ }^{(4)}$ não observaram nenhum caso. Em nosso estudo, foram descritos $2(18,1 \%)$ casos, ambos também apresentaram assimetria das alças (uma alça no sulco e a outra na pars plana). Loya et al. ${ }^{(6)}$ relataram que não houve relação estatisticamente significativa entre a inclinação da lente e a assimetria das alças em seu estudo.

Em nossa série de casos, a descentração da LIO foi encontrada em dois olhos implantados com a LIO de peça única do modelo SLIM ${ }^{\circledR}$ (Mediphacos Inc.), que não seria ideal para o posicionamento das alças no sulco ciliar, pois apresenta comprimento alça-a-alça inadequado. Outros 7 olhos da amostra não demonstraram descentração da LIO: 2 olhos com a LIO de peça única modelo SLIM (Mediphacos) e 5 olhos com LIO de três peças modelo TYPE7B ${ }^{\circledR}$ (Alcon, Inc.), que também não têm indicação para implante no sulco ciliar. Sugere-se que outros fatores possam contribuir para a descentração de uma LIO com alças posicionadas no sulco cililar e influenciar no resultado pós-operatório. O trabalho retrospectivo com avaliação dos prontuários não permitiu determinar informações relevantes como: tamanho da capsulorrexis, extensão da RCP (no pós-operatório imediato e no acompanhamento), quantidade de perda vítrea, descrição do procedimento de vitrectomia anterior.

Considerando-se o exame oftalmológico, poucas alterações foram encontradas na série. O seguimento pós-operatório prolongado é fundamental para a observação de complicações que podem vir a ocorrer após RCP e implante das alças da LIO no sulco ciliar. A média de seguimento dos pacientes do presente estudo foi de 103,09 \pm 32,93 dias, variando de 2 a 5 meses. A incidência de complicações relatada na presente amostra pode ser relacionada ao tempo de seguimento mais curto em relação a outros estudos com seguimento mais longo: 4 anos (variando entre 1 e 7 anos) $)^{(6)}$ e de 7 a 85 meses $^{(4)}$.

Em todos os casos foi observada uma hiperpigmentação do trabeculado, provavelmente em decorrência ao trauma da íris durante

Tabela 4. Achados do exame oftalmológico da última visita pós-operatória em olhos submetidos a implante de lente intraocular no sulco ciliar

\begin{tabular}{|c|c|c|c|c|c|c|c|c|}
\hline \multirow[b]{2}{*}{ Paciente } & \multicolumn{4}{|c|}{ Biomicroscopia } & \multirow{2}{*}{$\begin{array}{c}\text { Tonometria } \\
(\mathrm{mmHg})\end{array}$} & \multirow{2}{*}{$\begin{array}{c}\text { Gonioscopia } \\
\text { (pigmentação) }\end{array}$} & \multirow{2}{*}{$\begin{array}{c}\text { Fundoscopia } \\
\text { RP/EM }\end{array}$} & \multirow{2}{*}{$\begin{array}{c}\text { AV corrigida } \\
\text { pós-operatória }\end{array}$} \\
\hline & Flare/células & Alteração iriana & Depósito LIO & Descentração LIO & & & & \\
\hline 1 & Ausente & Ausente & Ausente & Ausente & 17 & $2+$ & Ausente/Ausente & $20 / 30$ \\
\hline 2 & Ausente & Presente & Presente & Ausente & 19 & $3+$ & Ausente/Ausente & $20 / 60$ \\
\hline 3 & Ausente & Presente & Ausente & Presente & 16 & $3+$ & Ausente/Ausente & $20 / 25$ \\
\hline 4 & Ausente & Ausente & Ausente & Ausente & 14 & $2+$ & Ausente/Ausente & $20 / 20$ \\
\hline 5 & Ausente & Ausente & Ausente & Ausente & 15 & $2+$ & Ausente/Ausente & $20 / 25$ \\
\hline 6 & Ausente & Presente & Ausente & Ausente & 12 & $2+$ & Ausente/Ausente & $20 / 30$ \\
\hline 7 & Ausente & Presente & Ausente & Ausente & 17 & $2+$ & Ausente/Ausente & $20 / 30$ \\
\hline 8 & Ausente & Presente & Ausente & Presente & 15 & $3+$ & Ausente/Ausente & $20 / 40$ \\
\hline 9 & Ausente & Ausente & Presente & Ausente & 18 & $2+$ & Ausente/Ausente & $20 / 30$ \\
\hline 10 & Ausente & Ausente & Ausente & Ausente & 16 & $2+$ & Ausente/Ausente & $20 / 20$ \\
\hline 11 & Ausente & Ausente & Ausente & Ausente & 13 & $2+$ & Ausente/Ausente & $20 / 60$ \\
\hline
\end{tabular}

$\mathrm{LIO}=$ lente intraocular; $\mathrm{RP}=$ rotura periférica de retina; $\mathrm{EM}=$ edema macular; $\mathrm{AV}=$ acuidade visual. 

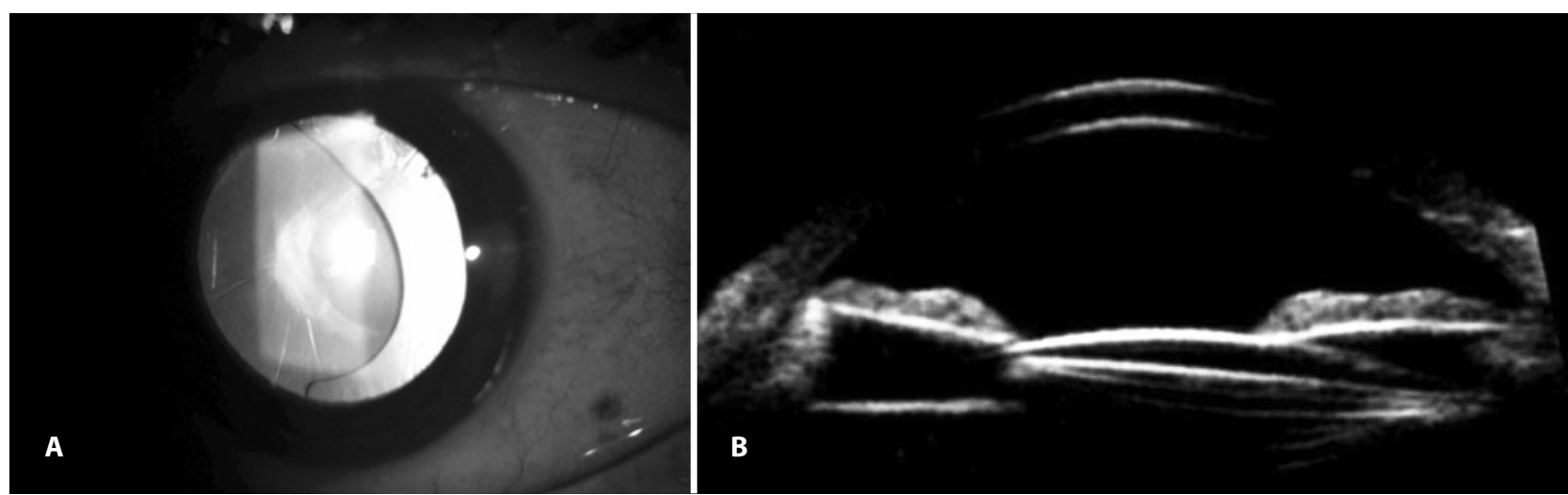

Figura 2. Olho 8 da série de olhos submetidos a implante intencional da lente intraocular no sulco ciliar durante cirurgia de catarata. A) Fotografia em lâmpada de fenda do olho esquerdo, em reflexo vermelho, sob midríase, demonstra a porção óptica da lente intraocular descentrada nasalmente, e também se observa a área da rotura de cápsula posterior. B) Biomicroscopia ultrassônica $(50 \mathrm{MHz})$ do mesmo olho demonstra, na varredura horizontal (meridianos 3 e 9 horas, representados à esquerda e à direita da imagem, respectivamente), o deslocamento da porção óptica da lente intraocular em direção ao meridiano de 9 horas (nasal).

a cirurgia e/ou pelo contato da LIO com a porção pupilar da íris, que foi observado no exame de UBM em 7 (63,6\%) olhos. Porém, não se observou defeitos de transiluminação de íris típicos do atrito entre a $\mathrm{LIO}$ e a íris. Talvez se estes olhos forem avaliados por um período mais longo, os defeitos irianos possam vir a ser identificados. As alterações irianas, observadas em $5(45,4 \%)$ olhos, mostraram-se características de lesões causadas pela manipulação excessiva durante a cirurgia, pois estavam localizadas nas regiões de entrada e saída dos instrumentos cirúrgicos através das incisões principal e acessória.

Somente um olho apresentou hipertensão ocular transitória após o procedimento cirúrgico, sendo a mesma controlada com medicação antiglaucomatosa (maleato de timolol 0,5\%) e, após seis semanas, houve normalização da PIO e a medicação foi suspensa. Nenhum caso de "flare" e/ou de células na câmara anterior foi observado. Vasavada et al.(4) também não observaram sinais de inflamação nos olhos estudados, e apenas um caso de glaucoma foi relatado. Também atentaram para a importância do monitoramento próximo nestes olhos, uma vez que o contato entre LIO e a superfície posterior da íris, principalmente nos implantes de LIO de peça única no sulco ciliar, pode causar inflamação crônica e maior risco de glaucoma.

Durante o exame de biomicroscopia em lâmpada-de-fenda, como o paciente está na posição sentada, muitas vezes o deslocamento posterior da LIO pode não ser observado ou então pode ser mascarado pela posição do paciente; o mesmo não ocorre no exame de UBM, uma vez que o paciente encontra-se deitado, sendo mais facilmente observado o deslocamento posterior da LIO. Portanto, nos casos de desestabilização do suporte capsular, o exame de UBM pode ser útil na avaliação do parâmetro de deslocamento posterior da LIO.

Estes achados permitem identificar os olhos candidatos a um acompanhamento seriado próximo para diagnóstico precoce de possíveis complicações relacionadas à técnica cirúrgica, como alteração da íris e do trabeculado, modificação gradual do posicionamento da $\mathrm{LIO}$ e desenvolvimento de hipertensão ocular.
Em conclusão, a biomicroscopia ultrassônica foi capaz de localizar as alças das lentes intraoculares implantadas intencionalmente no sulco ciliar em olhos com ruptura da cápsula posterior durante a cirurgia de catarata. Na amostra estudada, a técnica foi capaz de relacionar os casos de descentração da lente intraocular com a implantação assimétrica das alças.

\section{REFERÊNCIAS}

1. Collins JF, Gaster RN, Krol WF, Colling CL, Kirk GF, Smith TJ; Department of Veterans Affairs Cooperative Cataract Study. A comparison of anterior chamber and posterior chamber intraocular lenses after vitreous presentation during cataract surgery: the Department of Veterans Affairs Cooperative Cataract Study. Am J Ophthalmol. 2003; 136(1):1-9.

2. Yilmaz A, Baser Z, Yurdakul NS, Maden A. Posterior chamber lens implantation techniques in posterior capsular rupture. Eur J Ophthalmol. 2004;14(1):7-13.

3. Vianna Filho RC, Freitas L, Allemann N, Lima AL. Biomicroscopia ultra-sônica na avaliação da posição das lentes intraoculares em uma técnica de fixação escleral. Arq Bras Oftalmol. 2000;63(5):349-54.

4. Vasavada AR, Raj SM, Karve S, Vasavada V, Vasavada V, Theoulakis P. Retrospective ultrasound biomicroscopic analysis of single-piece sulcus-fixated acrylic intraocular lenses. J Cataract Refract Surg. 2010;36(5):771-7.

5. Chang DF, Masket S, Miller KM, Braga-Mele R, Little BC, Mamalis N, Oetting TA, Packer M; ASCRS Cataract Clinical Committee. Complications of sulcus placement of singlepiece acrylic intraocular lenses: recommendations for backup IOL implantation following posterior capsule rupture. J Cataract Refract Surg. 2009;35(8):1445-58.

6. Loya N, Lichter H, Barash D, Goldenberg-Cohen N, Strassmann E, Weinberger D. Posterior chamber intraocular lens implantation after capsular tear: ultrasound biomicroscopy evaluation. J Cataract Refract Surg. 2001;27(9):1423-7.

7. Amino K, Yamakawa R. Long-term results of out-of-the-bag intraocular lens implantation. J Cataract Refract Surg. 2000;26(2):266-70. Comment in J Cataract Refract Surg. 2000;26(8):1102-3.

8. Pavlin CJ, Harasiewicz K, Foster FS. Ultrasound biomicroscopic analysis of haptic position in late-onset, recurrent hyphema after posterior chamber lens implantation. J Cataract Refract Surg. 1994;20(2):182-5.

9. Ozdal PC, Mansour M, Deschênes J. Ultrasound biomicroscopy of pseudophakic eyes with chronic postoperative inflammation. J Cataract Refract Surg. 2003;29(6):1185-91. 